\title{
Guest Editorial: Nanostructured Thin Films
}

\author{
Geoff B. Smith \\ Department of Physics and Advanced Materials, University of Technology, Sydney \\ PO Box 123 Broadway, NSW 2007, Australia \\ g.smith@uts.edu.au
}

Nanostructured thin films (NSTF) are providing enormous scope for advances in optical, electronic and optoelectronic engineering and underpinning key developments in nanoscience. From a scientific perspective they embody numerous challenges and opportunities: covering concepts, models, growth, structure and characterization. Emerging technologies which depend on NSTF include photonics and plasmonics for information processing and communications; semiconductor and molecular electronics; display and lighting; single molecule bio-sensing, proteomics, advanced medical therapies, diagnostics and imaging; large scale, low cost approaches to energy efficiency, renewable energy and energy storage; and material surfaces which are durable, self-repair, self-clean, and have the ability to sense, change color, and to modulate reflectance, transmittance or thermal emittance.

Nanometal based composites are known from antiquity and featured as coatings in stunning luster's on Renaissance pottery [1]. Relevant nano-optics science dates back over a century and includes the classic 1857 paper by Faraday [2], and the resonant model of Maxwell Garnett (MG) [3], which is able to describe the spectral response of many of today's thin films, either in its original form or with various enhancements. For an excellent historical overview of the evolution of this subject from the middle of the $19^{\text {th }}$ century until the mid1990's I recommend the collection of facsimiles of landmark original papers put together by Lakhtakia [4]. Technological and scientific interest in spectral control using nano-composite cermet coatings blossomed during the oil crises in the 1970's for spectrally selective solar absorbers [5]. Gold black layers made much earlier [6] provided one interesting test for the emerging models in that period as the simple models such as (MG) could not handle touching or close nanoparticles. (Vacuum deposition at elevated pressure of gold black coatings exemplifies how early stage NSTF studies can lead to broader developments, in that case mass producing nanoparticles in vacuum). In the 1980's energy efficient spectrally selective windows based on nano-thin strongly plasmonic metal in insulator/metal/insulator multilayers evolved to become one of the first commodity scale high technology plasmonic thin film products. Dense silver films in these multilayers are produced today on the 60 million $\mathrm{m}^{2}$ per year scale at precision $\sim 11$ to $12 \mathrm{~nm}$ thicknesses with surprisingly low loss for such thicknesses (due to the specific adjacent dielectric layers used). Such multilayers have important lessons and roles for emerging NSTF work, as seen in two of the papers in this selection. Understanding growth in ultra thin noble metal layers was a necessary prelude to this technology and their transition in growth from isolated metal nano-islands, to conductors with nanovoids, to void free layers with nanostructured surfaces had already received much attention dating back to the 1930's [7] through to the 1970's [8] and 1980's for solar control windows [9]. New uses and insights are still emerging. In this edition improved plasmonic based sensing is demonstrated in two papers; with controlled nano-island shapes using special multilayers as both templates and for phase control by Suzuki et al., and with uses of ultra thin silver and gold layers plus a thin dielectric neighboring layer by Laval et al.. The latter exploits induced wave-guiding within the neighboring dielectric layer. The associated evanescent fields significantly enhance the metal layer's plasmonic response when under a prism. Coupling between polaritons and localized particle based surface plasmons across thin 
dielectrics is another way of achieving a similar goal and of emerging interest. Then the dense metal layer/dielectric interface acts as a surface plasmon polariton waveguide.

This is also an example of resonant energy transfer (RET) between nano-layers. It is a field of growing interest and is addressed very coherently and clearly in the paper by Bradshaw and Andrews who apply it to two close NSTF for all optical switching and signal processing based on forbidding spontaneous RET. This allows control via an auxiliary laser beam of switching energy between two layers of nanoparticles in a square array. Their quantum based formalism allows a thorough analysis of how the array structure influences the desired and undesired transfers of energy between individual elements in the array, in particular the key parameter the spacing between layers relative to the lattice spacing.

The percolation threshold in growing noble metal film or gradually densifying deposits of nanoparticles was of interest in the early thin metal film work noted above. A new aspect of the underlying physics behind this long saga was added in 2008 in blending data on Au on these 2D films with that of more complex 3D nanoporous layers made by etching alloys which exhibit hyper-dimensionality in their connectivity. A unified fractal model linked to the growth mechanism [10] emerges. Percolation of plasmonic material within an insulator can also develop if the film material undergoes a progressive insulator-metal phase transition. The paper by Gentle et al. in this set deals with that situation, with some surprising results. A full understanding of $\mathrm{VO}_{2}$ and its films will likely only happen if and when the high $\mathrm{T}_{\mathrm{c}}$ superconductor problem is solved (or vice versa) as the underlying physics seems to have much in common. $\mathrm{VO}_{2}$ is not a normal Fermi liquid in the metal phase, and as this paper shows not a normal semiconductor either at low temperatures. What happens in such highly correlated electron systems in nanoscale entities is a frontier of rich potential for future thin film studies.

Nanostructured insulating and semiconducting thin films also have a long and important history [11]. Their early deleterious impact on refractive index and electrical properties has been turned around to yield new approaches to NSTF, new science and applications; for example designed anisotropy and polarization control via oblique deposition and new sensing protocols. The paper by Suzuki et al. in this collection combines obliquely deposited dielectric layers with obliquely coated metal deposits. Ordered arrays of holes in thin layers of semiconductor open the door to exploiting shifts in 2D photonic crystal band gaps with infiltration of various species for sensing. The paper by Martin-Palma et al. in this edition shows convincingly that the band structure changes obtained by filling the holes in $\mathrm{Si}$ with porous silicon provides a useful chemical sensing modality. Sensitivity though is best if the chemical species of interest has a high index. Analysis of the latter utilized a 3-phase classical effective medium model. Effective medium or optical homogenization models of various complexities have become a mainstay of nanostructured thin film science and technology and feature in four of the papers in this edition. Sometimes however it is necessary or useful to go beyond quasi-static EM models to full wave analysis, which is usually the case in photonic crystal arrays. Simple changes in the 2D photonic crystal structure is elegantly considered using Rigorous Coupled Wave (RCW) theory within a multilayer high/low index stack carrying the hole array. Multi-narrow band optical filtering in this paper by Shambat et al. is achieved compactly by varying the hole diameter in close-by arrays. The impact of practical influences arising in fabrication are also modeled in this paper resulting in design guidelines for an important advance in diagnostics directed at enhanced detection of incipient retinal disease.

1D and 2D photonic crystal arrays feature in the novel approach to optical homogenization in the paper by the Felbacq group. Their approach does not have to invoke the quasi-static limit. It is in effect a scaling model and provides a way of linking the average macroscopic fields to those at the nanoscale by introducing a hidden spatial variable. The comparison of spectral reflectance and transmittance found using this homogenization approach to results found with a full wave treatment for the grating structures evaluated is quite convincing. 
Apart from accuracy the computational effort required is an important discriminating issue between homogenization models. This approach does not seem to be computationally onerous.

As with most NSTF optical work these days all of the papers in this group presenting data needed to invoke nanostructure control via specially refined and controlled deposition protocols, sometimes relying on templates. Otherwise post deposition patterning using nanoscale lithography or ion beam and chemical etching techniques to yield both ordered and semi-random nanostructures were used. Accurate geometric characterization of samples at the nanoscale, preferably in all 3-dimensions, is important in getting reliably to grips with the structure-composition-properties link. Fitting optical data with generic mathematical models to find sample parameters can be unreliable and should be backed up with direct methods where possible. Many excellent methods and probes are now available for this task, though the high resolution SEM continues to be the mainstay. Theoretical modeling in this field has until quite recent times tended to be well ahead of the available sample structures and the experimental probes needed to test models. Its main imitation has probably been computational effort and a focus on ordered arrays as in photonic crystals. The majority of data however until this century has been in the far field on samples with random or partly ordered nanostructure. The electromagnetic models most suited to that situation have tended to be the classical approximate ones, with no multipoles. Now procedures for making large enough ordered arrays are well established, and various tools exist for probing spectral properties at the nanoscale, even for a very small cluster of two or three nanoparticles, or for exploring plasmon localization effects as in nanoholes or partly random structures. Thus experiment has now largely caught up, and much opportunity exists using the interplay between experiment and theory for significant progress in this field to help meet the significant technological needs and challenges of this century.

\section{Acknowledgments}

I wish to thank all the authors for contributing to this special section on Nanostructured Thin Films and also those that participated in the associated SPIE Optics and Photonics Symposium and NSTF conference in 2008 and contributed to its valuable Proceedings.

\section{References}

[1] S. Padovani, C. Sada, P. Mazzoldi, B. Brunetti, I. Borgia, A. Sgamelloti, A. Giulivi, and F. D'Acapito, "Copper in glazes of Renaissance luster pottery: nanoparticles, ions and local environment," J. Appl. Phys. 93, 10058-10063 (2003) [doi:10.1063/1.1571965].

[2] M. Faraday, "Experimental relations of gold (and other metals) to light," Philos. Trans. Soc. Lond. 145, 147-181 (1857).

[3] J. C. Maxwell Garnett, "Colours in metal glasses and in metallic films," Philos. Trans. Soc. Lond. A 203, 385-420 (1904) [doi:10.1098/rsta.1904.0024]

[4] A. Lakhtakia, Ed., Selected Papers on Linear Optical Composite Materials, SPIE Milestone Series, vol. MS 120, SPIE, Bellingham, WA (1996).

[5] G. A. Niklasson and C. G. Granqvist, "Surfaces for selective absorption of solar energy: an annotated bibliography 1955-1981," J. Mater. Sci. 18, 3475-3534 (1983) [doi:10.1007/BF00540724].

[6] L. Harris, R.T. McGinnies, and B. M. Siegel, "The preparation and optical properties of gold blacks," J. Opt. Soc. Am. 38, 582-589 (1948) [doi:10.1364/JOSA.38.000582].

[7] E. David, "Deutung der Anomalien der optischen Konstanten dünner Metallschichten," Z. Phys. 114, 389-406 (1939) [doi: 10.1007/BF01329519]. 
[8] D. N. Jarrett and L. Ward, "Optical properties of discontinuous gold films," J. Phys. D:Appl. Phys. 9, 1515-1527 (1976) [doi: 10.1088/0022-3727/9/10/017].

[9] G. B. Smith, G. A. Niklasson, J. S. E. M. Svensson, and C. G. Granqvist, "Noble metal-based transparent infrared reflectors: experiments and theoretical analyses for very thin gold films," J. Appl. Phys. 59, 571-581 (1986) [doi:10.1063/1.336615].

[10] G. B. Smith, A.I. Maaroof, and M.B. Cortie, "Percolation in nanoporous gold and the Principle of Universality from two dimensions to hyperdimensions," Phys. Rev B. 78, 165418 (2008) [doi: 10.1103/PhysRevB.78.16518].

[11] A. Lakhtakia and R. F. Messier, Eds., Engineered Nanostructural Films and Materials, SPIE, Bellingham, WA(1999). 